\title{
Approximation Algorithms for Scheduling with Rejection on Two Unrelated Parallel Machines
}

\author{
Feng Lin, Xianzhao Zhang*, Zengxia Cai \\ College of Science \\ Linyi University \\ Linyi, Shandong, China 276005
}

\begin{abstract}
In this paper, we study the scheduling problem with rejection on two unrelated parallel machines. We may choose to reject some jobs, thus incurring the corresponding penalty. The goal is to minimize the makespan plus the sum of the penalties of the rejected jobs. We first formulate this scheduling problem into an integer program, then relax it into a linear program. From the optimal solution to the linear program, we obtain the two algorithms using the technique of linear programming rounding. In conclusion, we present a deterministic 3-approximation algorithm and a randomized 3approximation algorithm for this problem.
\end{abstract}

Keywords-Scheduling; Rejection; Approximation algorithm; Linear programming; Rounding

\section{INTRODUCTION}

The unrelated parallel machine scheduling problem to minimize makespan, $R \| C_{\max }$ following the notation of Graham et al. [1], is one of the classic NP- hard problems in combinatorial optimization. This problem is mentioned in many works concerning approximation algorithms [2,3], and it has received much attention in the past few decades with many extant approximation algorithms, among which the currently best 2-approximation algorithm is due to [4], who also show that the problem does not admit an algorithm with approximation ratio smaller than $3 / 2$, unless $P=N P$. One special case for this problem-Each job can only be assigned to a subset of the machine set with the same processing timewhich is also known as the restricted assignment problem. Does there exist an approximation algorithm with approximation ratio better than 2 ? This problem is regarded as one of the ten open problems in combinatorial optimization [3].

The scheduling problem with rejection arises in make-toorder production systems with limited production capacity and tight delivery requirements, where simultaneous job rejection and scheduling decisions have to be made for maximizing the total revenue. In such systems, accepting orders without considering their impact on the whole system may delay some of the orders beyond their due dates. To be able to preserve the high quality of service to customers of accepted orders, the manufacturer has to determine which orders to accept and how to schedule them to maximize total revenue.

Many important results concerning the parallel machine scheduling problems with rejection appear in recent years. In reference [5] Engels et al. develop some techniques to design approximation algorithm for the general problem with rejection. Their main technique is to reduce a problem with rejection to a scheduling problem without rejection based on the linear programming rounding method. Hoogeveen, Skutella and Woeginger [6] consider the preemptive scheduling with rejection, and the goal is to optimize the preemptive makespan on the $m$ parallel machines plus the sum of the penalties of the rejected jobs. They provide a complete classification of these scheduling problems on complexity and approximability. On the variant with an arbitrary number of unrelated machines, which is APX-hard, they propose a 1.58-approximation algorithm for it. Moreover, their results for unrelated machines may be carried over to the corresponding preemptive open shop scheduling problem with rejection. Li and Yuan [7] consider several parallel machine scheduling problems with deteriorating jobs and rejection. The objective is to minimize the scheduling cost of the accepted jobs plus The total penalty of the rejected jobs. They propose two fully polynomial time approximation schemes for the problems under consideration. In reference [8] Gerstl and Mosheiov study scheduling problems with rejection and general position-dependent processing times on identical parallel machines, and they introduce efficient algorithms for the problems, which run in $O\left(n^{m+3}\right)$ time (which is polynomial for a given number of machines). There are many other important results ([9-13]).

In this paper, we study the following problem: We have two machines $\left\{M_{1}, M_{2}\right\}$, together with a job set $\left\{J_{1}, \cdots, J_{n}\right\}$. The processing time of job $J_{j}$ on machine $M_{i}$ is $p_{i j}(i=1,2)$. We may choose not to process job $J_{j}$, thus incurring a penalty $q_{j}$. The objective is to optimize the makespan for the processed jobs plus the sum of the penalties of the rejected jobs. If we denote by $J_{A}$ the set of processed jobs, and $J_{R}$ the set of rejected jobs, the problem may be expressed as $R_{2}|r e j| C_{\max }\left(J_{A}\right)+\sum_{j \in J_{R}} q_{j}$.

We organize this paper as follows: In section Two we give a deterministic 3-approximation algorithm for the problem under consideration. In section Three a randomized 3approximation algorithm is presented for this problem. In section Four we give some concluding remarks. 


\section{A 3-APPROXIMATION ALGORITHM FOR}

$$
R_{2}|r e j| C_{\max }\left(J_{A}\right)+\sum_{j \in J_{R}} q_{j}
$$

We introduce a decision variable $z_{j}$ for job $J_{j}(j=1,2, \cdots, n)$, with the following meaning:

$$
z_{j}=\left\{\begin{array}{l}
1, i f-j o b-J_{j}-i s-\text { rejected } \\
0, \text { else }
\end{array}\right.
$$

For each machine-job pair $(i, j)(i=1,2 ; j=1,2, \cdots, n)$, we introduce a decision variable $x_{i j}$ with the following meaning:

$$
x_{i j}=\left\{\begin{array}{l}
1, \text { if }- \text { job }-J_{j}-i s-\text { processed }- \text { on }-M_{i} \\
0, \text { otherwise }
\end{array}\right.
$$

Based on the above notations we formulate the scheduling problem into an integer program.

$$
\begin{array}{ll}
\min & T+\sum_{j=1}^{n} q_{j} z_{j} \\
\text { s.t. } \quad & x_{1 j}+x_{2 j}+z_{j}=1, \quad j=1,2, \cdots, n \\
& \sum_{j=1}^{n} p_{1 j} x_{1 j} \leq T, \\
& \sum_{j=1}^{n} p_{2 j} x_{2 j} \leq T, \\
& x_{i j} \in\{0,1\}, \quad i=1,2 ; j=1,2, \cdots, n \\
& z_{j} \in\{0,1\}, \quad j=1,2, \cdots, n .
\end{array}
$$

We first relax integer program (1) into the following linear program.

$$
\begin{array}{ll}
\min & T+\sum_{j=1}^{n} q_{j} z_{j} \\
\text { s.t. } \quad & x_{1 j}+x_{2 j}+z_{j}=1, \quad j=1,2, \cdots, n \\
& \sum_{j=1}^{n} p_{1 j} x_{1 j} \leq T, \\
& \sum_{j=1}^{n} p_{2 j} x_{2 j} \leq T, \\
& x_{i j} \geq 0, \quad i=1,2 ; j=1,2, \cdots, n \\
& z_{j} \geq 0, \quad j=1,2, \cdots, n .
\end{array}
$$

We denote by $\left(x_{1 j}^{*}, x_{2 j}^{*}, z_{j}^{*}\right)$ the optimal solution to linear program (2). Obviously we have $T^{*}+\sum_{j=1}^{n} q_{j} z_{j}^{*} \leq O P T$.
Here $T^{*}=\max \left\{\sum_{j=1}^{n} p_{1 j} x_{1 j}^{*}, \sum_{j=1}^{n} p_{2 j} x_{2 j}^{*}\right\}, O P T$ stands for the optimal objective value for the scheduling problem.

We apply the following rounding procedure to $\left(x_{1 j}^{*}, x_{2 j}^{*}, z_{j}^{*}\right)$.

\section{Rounding Procedure $R_{1}$}

Step 1: Whenever $z_{j}^{*} \geq \alpha \quad(0<\alpha<1)$, set $\bar{z}_{j}=1_{\text {(job }}$ $J_{j}$ is rejected).

Step 2: Otherwise set $\bar{z}_{j}=0$ (job $J_{j}$ is processed).

Step 3: Whenever $x_{1 j}^{*} \geq x_{2 j}^{*}$, set $\bar{x}_{1 j}=1$. (job $J_{j}$ is processed on machine $M_{1}$ ).

Step 4: Else set $\bar{x}_{2 j}=1$. (job $J_{j}$ is processed on machine $M_{2}$ ).

We now analyze the quality of the solution obtained by Rounding Procedure $R_{1}$.

We first take a look at the makespan $\bar{T}$, here $\bar{T}=\max \left\{\bar{L}_{1}, \bar{L}_{2}\right\}$.

$$
\begin{gathered}
\bar{L}_{1}=\sum_{j: \bar{x}_{1 j}=1} p_{1 j}=\sum_{j: x_{1 j}^{*}>\frac{1-\alpha}{2}} p_{1 j}<\frac{2}{1-\alpha} \sum_{j: x_{1 j}^{*}>\frac{1-\alpha}{2}} p_{1 j} x_{1 j}^{*} \\
\leq \frac{2}{1-\alpha} \sum_{j=1}^{n} p_{1 j} x_{1 j}^{*} \leq \frac{2}{1-\alpha} T^{*} .
\end{gathered}
$$

Similarly we have $\bar{L}_{2} \leq \frac{2}{1-\alpha} T^{*}$. Therefore we get $\bar{T} \leq \frac{2}{1-\alpha} T^{*}$

As for the total penalty for the rejected jobs, we have

$$
\sum_{j: z_{j}=1} q_{j}=\sum_{j: z_{j}^{*} \geq \alpha} q_{j} \leq \frac{1}{\alpha} \sum_{j: z_{j}^{*} \geq \alpha} q_{j} z_{j}^{*} \leq \frac{1}{\alpha} \sum_{j=1}^{n} q_{j} z_{j}^{*}
$$

So we have

$$
\begin{gathered}
\bar{T}+\sum_{j: z_{j}=1} q_{j} \leq \frac{2}{1-\alpha} T^{*}+\frac{1}{\alpha} \sum_{j=1}^{n} q_{j} z_{j}^{*} \\
\leq f(\alpha)\left(T^{*}+\sum_{j=1}^{n} q_{j} z_{j}^{*}\right) \leq f(\alpha) O P T .
\end{gathered}
$$


Here $f(\alpha)=\max \left\{\frac{2}{1-\alpha}, \frac{1}{\alpha}\right\}$ is the approximation ratio. The value for $f(\alpha)$ is minimized if and only if $\frac{2}{1-\alpha}=\frac{1}{\alpha}$, i.e., $\alpha=\frac{1}{3}$, the minimum value for $f(\alpha)$ is 3 .

Based on the discussions above we propose a 3approximation algorithm for $R_{2}|r e j| C_{\max }\left(J_{A}\right)+\sum_{j \in J_{R}} q_{j}$.

\section{Algorithm 1}

Step 1: Formulate the scheduling problem into an integer program (1).

Step 2: Relax integer program (1) to linear program (2).

Step 3: Solve linear program (2) and obtain an optimal solution $\left(x_{1 j}^{*}, x_{2 j}^{*}, z_{j}^{*}\right)$.

$$
\begin{aligned}
& J_{R}=\left\{j: z_{j}^{*} \geq \frac{1}{3}\right\} J_{A}=\left\{j: z_{j}^{*}<\frac{1}{3}\right\} \\
& \text { Step 4: Set } \\
& J_{M_{1}}=\left\{j: x_{1 j}^{*} \geq x_{2 j}^{*}, z_{j}^{*}<\frac{1}{3}\right\} \\
& J_{M_{2}}=\left\{j: x_{2 j}^{*} \geq x_{1 j}^{*}, z_{j}^{*}<\frac{1}{3}\right\} .
\end{aligned}
$$

Step 6: Process the jobs in $J_{M_{i}}(i=1,2)$ continuously on machine $M_{i}$ in an arbitrary order.

Theorem 1. Algorithm 1 is a 3-approximation algorithm for $R_{2}|r e j| C_{\max }\left(J_{A}\right)+\sum_{j \in J_{R}} q_{j}$.

\section{A RANDOMIZED 3-APPROXIMATION ALGORITHM FOR$$
R_{2}|r e j| C_{\max }\left(J_{A}\right)+\sum_{j \in J_{R}} q_{j}
$$

In section Two we have a deterministic principle that determines the set $J_{R}, J_{M_{1}}$ and $J_{M_{2}}$. In this section the processed jobs are assigned to machine $M_{1}, M_{2}$ with some probability.

We still use program (2) as a linear program relaxation for the scheduling problem. For the optimal solution $\left(x_{1 j}^{*}, x_{2 j}^{*}, z_{j}^{*}\right)$ to linear program (2), we apply the following rounding procedure.

$$
\text { Rounding Procedure } R_{2}
$$

Step 1: Whenever $z_{j}^{*} \geq \alpha \quad(0<\alpha<1)$, set $\bar{z}_{j}=1$ (job $J_{j}$ is rejected)

Step 2: Otherwise set $\bar{z}_{j}=0{ }_{(j o b} J_{j}$ is processed). Job $J_{j}$ is assigned to machine $M_{i}(i=1,2)$ with probability $\tilde{x}_{i j}=\frac{x_{i j}^{*}}{x_{1 j}^{*}+x_{2 j}^{*}}$.

In rounding procedure $R_{2}$, the set of rejected jobs $J_{R}$ is a deterministic set. While the schedule formed by the jobs in $J_{A}$ is a randomized one. We denote by $L_{i}(i=1,2)$ the load on machine $M_{i}$ in the randomized schedule, and by $T$ the makespan for the schedule. Obviously we have $T=\max \left\{L_{1}, L_{2}\right\}$.

For processed job ${ }^{J_{j}}$, obviously we have $z_{j}^{*}<\alpha$, $x_{1 j}^{*}+x_{2 j}^{*}>1-\alpha$

$$
\begin{aligned}
\tilde{x}_{i j}=\frac{x_{i j}^{*}}{x_{1 j}^{*}+x_{2 j}^{*}} & <\frac{x_{i j}^{*}}{1-\alpha} . \\
E\left[L_{1}\right] & =\sum_{j: z_{j}^{*}<\alpha} p_{1 j} \tilde{x}_{1 j}<\frac{1}{1-\alpha} \sum_{j: z_{j}^{*}<\alpha} p_{1 j} x_{1 j}^{*} \\
\leq & \frac{1}{1-\alpha} \sum_{j=1}^{n} p_{1 j} x_{1 j}^{*} \leq \frac{1}{1-\alpha} T^{*}
\end{aligned}
$$

Similarly we have $E\left[L_{2}\right] \leq \frac{1}{1-\alpha} T^{*}$.

Lemma 1. $E[T] \leq E\left[L_{1}\right]+E\left[L_{2}\right]$.

Proof: $\forall J_{M_{1}} \subseteq J_{A}$.

The notation $\left(J_{M_{1}}, J_{A} \backslash J_{M_{1}}\right)$ stands for the random event that jobs in $J_{M_{1}}$ are assigned to machine $M_{1}$, while the jobs in $J_{A} \backslash J_{M_{1}}$ are assigned to machine $M_{2}$.

Obviously we have

$$
\begin{gathered}
P_{r}\left(J_{M_{1}}, J_{A} \backslash J_{M_{1}}\right)=\prod_{j: j \in J_{M_{1}}} \tilde{x}_{1 j} \prod_{j: j \in J_{A} \backslash J_{M_{1}}} \tilde{x}_{2 j} \\
L_{1}\left(J_{M_{1}}, J_{A} \backslash J_{M_{1}}\right)=\sum_{j: j \in J_{M_{1}}} p_{1 j}
\end{gathered}
$$




$$
\begin{aligned}
& L_{2}\left(J_{M_{1}}, J_{A} \backslash J_{M_{1}}\right)=\sum_{j: j \in J_{A} \backslash J_{M_{1}}} p_{2 j} \\
& E\left[L_{1}\right]=\sum_{J_{M_{1}} \subseteq J_{A}}\left(\sum_{j: j \in J_{M_{1}}} p_{1 j}\right) P_{r}\left(J_{M_{1}}, J_{A} \backslash J_{M_{1}}\right) \\
& E\left[L_{2}\right]=\sum_{J_{M_{1}} \subseteq J_{A}}\left(\sum_{j: j \in J_{A} \backslash J_{M_{1}}} p_{2 j}\right) P_{r}\left(J_{M_{1}}, J_{A} \backslash J_{M_{1}}\right) \\
& E[T]= \\
& \sum_{J_{M_{1}} \subseteq J_{A}} \max \left\{\sum_{j: j \in J_{M_{1}}} p_{1 j}, \sum_{j: j \in J_{A} \backslash J_{M_{1}}} p_{2 j}\right\} P_{r}\left(J_{M_{1}}, J_{A} \backslash J_{M_{1}}\right)
\end{aligned}
$$

Obviously we have $E[T] \leq E\left[L_{1}\right]+E\left[L_{2}\right]$. The proof is completed.

From the discussions above we have $E[T] \leq \frac{2}{1-\alpha} T^{*}$.

As mentioned in section 2, we have an upper bound for the total penalty of the rejected jobs

$$
\sum_{j: z_{j}=1} q_{j}=\sum_{j: z_{j}^{*} \geq \alpha} q_{j} \leq \frac{1}{\alpha} \sum_{j: z_{j}^{*} \geq \alpha} q_{j} z_{j}^{*} \leq \frac{1}{\alpha} \sum_{j=1}^{n} q_{j} z_{j}^{*}
$$

Hence we have

$$
\begin{aligned}
E[T]+ & \sum_{j: \bar{z}_{j}=1} q_{j} \leq \frac{2}{1-\alpha} T^{*}+\frac{1}{\alpha} \sum_{j=1}^{n} q_{j} z_{j}^{*} \\
& \leq \max \left\{\frac{2}{1-\alpha}, \frac{1}{\alpha}\right\} O P T
\end{aligned}
$$

The approximation ratio $\max \left\{\frac{2}{1-\alpha}, \frac{1}{\alpha}\right\}$ is minimized when $\alpha=\frac{1}{3}$, and the minimum value is 3 .

We give a randomized 3-approximation algorithm for $R_{2}|r e j| C_{\max }\left(J_{A}\right)+\sum_{j \in J_{R}} q_{j}$ in the following way.

\section{Algorithm 2}

Step 1: Formulate the scheduling problem into an integer program (1).

Step 2: Relax integer program (1) to linear program (2).

Step 3: Solve linear program (2) and obtain an optimal solution $\left(x_{1 j}^{*}, x_{2 j}^{*}, z_{j}^{*}\right)$.
Step 4: Set $J_{R}=\left\{j: z_{j}^{*} \geq \frac{1}{3}\right\}, J_{A}=\left\{j: z_{j}^{*}<\frac{1}{3}\right\}$.

Step 5: For job j satisfying $z_{j}^{*}<\frac{1}{3}$, assign job $j$ to machine $M_{i}(i=1,2)$ with probability $\tilde{x}_{i j}=\frac{x_{i j}^{*}}{x_{1 j}^{*}+x_{2 j}^{*}}$.

Step 6: Process the jobs in $J_{M_{i}}(i=1,2)$ continuously on machine $M_{i}$ in an arbitrary order.

Theorem 2. Algorithm 2 is a randomized 3-approximation algorithm for $R_{2}|r e j| C_{\max }\left(J_{A}\right)+\sum_{j \in J_{R}} q_{j}$.

\section{CONCLUSION}

In this paper, we study the scheduling problem with rejection on two unrelated parallel machines. We may choose not to process some jobs, thus incurring the corresponding penalty. The goal is to minimize the makespan plus the sum of the penalties of the rejected jobs. We present a deterministic 3approximation algorithm and a randomized 3-approximation algorithm for this problem. We obtain the two algorithms using the technique of linear programming rounding.

\section{ACKNOWLEDGMENT}

The authors are thankful to the reviewers for their valuable suggestions. This work was supported by the Chinese Society of Logistics and the China Federation of Logistics and Purchasing Project (2015CSLKT3-199), the Logistics Teaching and Research Reformation Projects for Chinese Universities (JZW2014048, JZW2014049), the Applied Mathematics Enhancement Program of Linyi University, and the national college students' innovation and entrepreneurship training program (201410452004).

\section{REFERENCES}

[1] R. L. Graham, E. L. Lawler, J. K. Lenstra and A. H. G. Rinnooy Kan, "Optimization and approximation in deterministic sequencing and scheduling: A survey", Annals of Discrete Mathematics, vol. 5, pp. 287326, 1979.

[2] V. V. Vazirani, Approximation Algorithms, Springer, Berlin, 2003.

[3] D. P. Williamson, D. B. Shmoys, The Design of Approximation Algorithms, Cambridge University Press, London, 2011.

[4] J. K. Lenstra, D. B. Shmoys and E. Tardos, "Approximation algorithms for scheduling unrelated parallel machines", Mathematical Programming, vol. 46, pp. 259-271, 1990.

[5] D. W. Engels, D. R. Karger, S. G. Kolliopoulos, S. Sengupta, R. N. Uma and J. Wein, "Techniques for scheduling with rejection", Journal of Algorithms, vol. 49, pp. 175-191, 2003.

[6] H. Hoogeveen, M. Skutella and G. J. Woeginger, "Preemptive scheduling with rejection", Mathematical Programming Ser B, vol. 94, pp. 361-374, 2003.

[7] S. S. Li and J. J. Yuan, "Parallel-machine scheduling with deteriorating jobs and rejection", Theoretical Computer Science, vol. 411, pp. 3642$3650,2010$.

[8] E. Gerstl and G. Mosheiov, "Scheduling on parallel identical machines with job-rejection and position-dependent processing times", Information Processing Letters, vol. 112, pp. 743-747, 2012. 
[9] D. Shabtay, N. Gaspar and M. Kaspi, "A survey on offline scheduling with rejection", Journal of Scheduling, vol. 16, pp. 3-28, 2013.

[10] L. Epstein and H. Z. Haider, "Online scheduling with rejection and withdrawal", Theoretical Computer Science, vol. 412, pp. 6666-6674, 2011.

[11] L. Epstein and H. Z. Haider, "Preemptive online scheduling with rejection of unit jobs on two uniformly related machines", Journal of Scheduling, vol. 17, pp. 87-93, 2014.
[12] L. F. Lu, C. T. Ng and L. Q. Zhang, "Optimal algorithms for singlemachine scheduling with rejection to minimize the makespan", International Journal of Production Economics, vol. 130, pp. 153-158, 2011.

[13] X. Min, Y. Q. Wang, J. Liu and M. Jiang, "Semi-online scheduling on two identical machines with rejection", Journal of Combinatorial Optimization, vol. 26, pp. 472-479, 2013. 